Current Research in Psychology 1 (1): 53-60, 2010

ISSN 1949-0178

(C) 2010 Science Publications

\title{
Olfaction and Emotion Content Effects for Memory of Vignettes
}

\author{
${ }^{1}$ Jeremy W. Grabbe, ${ }^{2}$ Ann L. McCarthy, ${ }^{3}$ Charissa M. Brown and ${ }^{3}$ Arlene A. Sabo \\ ${ }^{1}$ Department of Psychology, Plattsburgh State University, \\ SUNY Plattsburgh, 101 Broad Street, Plattsburgh, NY 12901 \\ ${ }^{2}$ Department of Psychology, The University of Akron, OH, USA \\ ${ }^{3}$ Department of Psychology, Plattsburgh State University, NY, USA
}

\begin{abstract}
Problem statement: Recent research has shown a relationship between olfaction and episodic/autobiographical memory. The mnemonic theory of odor asserts that odor representation and storage is tied to memory. The Proust phenomenon suggests that specifically episodic memory is the memory component behind the mnemonic theory of olfaction. Neurological evidence demonstrates that neural structures related to emotion have connections between olfactory receptors and the episodic memory center. Approach: This study examined the role of emotion and olfaction in memory for vignettes. Participants were presented with a series of vignettes that varied by emotional content. Olfactory cues were paired with vignettes. Participants were questioned over recall of the vignettes. Results: Two experiments demonstrated a significant effect for emotion in memory performance for vignettes. The role of olfaction was not as prominent. Conclusion/Recommendations: This confirms the Proust phenomenon olfaction, namely that olfaction plays a greater role in autobiographical memory than memory for vignettes. The generalization of the Proust phenomenon to nonautobiographical memory is not supported by the results of these two studies. The authors suggest future research examining the interaction between olfaction and emotion should be directed towards autobiographical memory.
\end{abstract}

Key words: Olfaction, nonautobiographical memory, sensory cues

\section{INTRODUCTION}

Olfaction and emotion content for vignettes: Olfaction is often overlooked in regards to its role in memory and cognition compared to intense scientific focus on vision and audition. Olfaction has been studied detecting, identifying, differentiating and remembering various odors (Jehl et al., 1995). The value of olfaction and olfaction research has increased as we have come to understand its role in everyday functioning. The role of olfaction in higher level cognitive processes is a crucial area that has been neglected. A new perspective on olfactory research now looks at how olfaction is involved in memory (Stevenson and Boakes, 2003) and has caused renewed interest in olfaction's functions in higher level processes.

Olfaction's importance has lead to many unique ways of measuring and studying our sense of smell. Olfaction is studied using many different measures. Some of these indexes are the University of Pennsylvania smell identification test, single ascending series butanol odor detection threshold test, odor recognition memory test and the Toyota and Takagi olfactometer. The psychometric soundness of several tests of olfactory ability has been studied (Doty et al., 1994). The researchers went on to conclude that some rather distinct tests share a common variance of olfactory ability. This corpus of research demonstrates the diversity of olfaction and its importance in modern psychological research as well as everyday functions. In this experiment, participants self-rated reaction (like, neutral, dislike) to olfactory stimuli was studied.

The process of olfaction: The uniqueness of the neural architecture of olfaction lends further credence to notions that olfaction deserves greater research focus than it has in the past. There are two different pathways for olfaction, one of which bypasses the thalamus via the limbic system. This second pathway will be of profound interest in this study.

Olfaction begins in the nasal cavity where olfactory receptors in a nasal layer called the olfactory mucosa interact with olfactory stimuli (Ganong, 1999). Axons from the olfactory mucosa send signals to the olfactory

Corresponding Author: Jeremy W. Grabbe, Department of Psychology, Plattsburgh State University, SUNY Plattsburgh, 101 Broad Street, Plattsburgh, NY 12901 
bulb which forms the first cranial nerve. The olfactory bulb transmits information to the amygdala and the piriform cortex. These areas are the first cortical regions to receive the olfactory information. This contributes to the uniqueness of olfaction in that it is the only sense to not directly transmit information through the thalamus to the cerebral cortex (Pinel, 1990). Only after going through the amygdala and the piriform cortex does the olfactory signal split into two pathways. One projects into the medial dorsal nuclei of the thalamus while the other pathway projects into the limbic system (Pinel, 1990). The pathway into the thalamus then leads to the hypothalamus and is then projected into the prefrontal cortex. The thalamus relays sensory information to the cortex and its substructure, the dorsomedial nucleus, is responsible for the consolidation of memories (Pinel, 1990). The second olfactory pathway that leads from the amygdala and the piriform cortex to the limbic system is of paramount importance during the course of this study. This projection is the neurological key to the hypothesis between olfaction, emotion and episodic memory.

Episodic memory: Why is olfaction such a powerful cue for retrieval for autobiographical episodic memories? There is a large amount of complexity for olfaction (Brand et al., 2001). There has been evidence that there are several different subsystems for olfaction, in fact sniffing and smelling are two distinct processes and even produce different patterns of cortical activation occurring for different hemispheres (Yamaguchi et al., 2001). This can allow an olfactory stimulus to be encoded simultaneously into different olfactory subsystems and thus create a redundancy in memory allowing odor memory to remain intact over time because of redundancy. This process is conceptually similar to Paivio (1971) dual-coding hypothesis. The dual-coding hypothesis states that learning (encoding) is enhanced when a stimulus can be represented in two different modalities. Episodic memory performance benefits from using olfaction as a sensory modality for cued recall.

The nature of episodic memory is centered on the context of the situation in which the memory is encoded (Malmberg and Shiffrin, 2005; Tulving, 1974). This context, provided by sensory information, provides a "tag" for to-be-remembered experiences so that they can be retrieved from long-term memory. For example, episodic memories that people recall often have descriptions of not only the things they saw or heard at that moment, but also vivid details from the other senses, such as certain smells that were present at the time of encoding (Malmberg and Shiffrin, 2005). In the case of flashbulb memories, there are often high levels of sensory detail (Talarico and Rubin, 2007). The presence of a cue at recall that was also present at the time of encoding can facilitate recall of the memory (Tulving, 1974). An important implication to the study of memory is that olfaction plays a large role as a sensory context cue in episodic memory (Stevenson and Boakes, 2003).

Olfaction and episodic memory: Olfaction's role in memory has been of great interest to researchers (Brand et al., 2001; Engen and Ross, 1973). Jehl et al. (1995) examined the relationship between familiarity with odors and recognition memory. Evidence suggests that odors that were previously presented allowed the subject to become more familiar with them. These same odors were far more discernable than odors that have not been familiarized. Jehl et al. (1995) exposed one group to various odors and another group was not familiarized with any odors. The researchers then conducted an odor discrimination task. Using signal detection theory, they found no significant difference in familiarity in the two groups when examining subjects' ability to correctly identify or discriminate an odor. While there was no significant difference between groups for hits, there was a significant difference in false alarms. The results demonstrated that the familiarized group displayed fewer false alarms than the unfamiliar group. Familiarization helped people inhibit false alarms for an odor that they had not encountered earlier, thus helping to improve recognition memory performance.

There is also evidence that the benefit olfactory cues have for recognition are resistant to forgetting over time. Engen and Ross (1973) demonstrated that, after a sharp drop off immediately after learning, olfaction has a shallower forgetting curve over time. Odors were found to still be significantly recognizable even after three months and this effect was even found for novel odors (odors never encountered before the study). This lends credence to the idea of the Proust phenomenon of olfaction (Chu and Downes, 2000) or Proustian memory (Parker et al., 2001). The Proust phenomenon of olfaction theorizes that autobiographical memories can be cued by an odor encountered only once before and many years prior. A study of autobiographical memories has found evidence that a cue can trigger autobiographical memories for their subjects (Chu and Downes, 2000).

The mnemonic theory of odor put forth by Stevenson and Boakes (2003) proposes that many of the more neuropsychological theories of odor perception have been falling short or found lacking in evidence for 
various chemical theories. They argued that the psychological aspects of odor perception have been largely ignored. According to Stevenson and Boakes (2003) theory, olfaction relies more heavily on experience than the other senses do (such as hearing and vision). In addition, there seems to be a greater similarity or relation between episodic memory and olfaction than previous research suggested. Indeed, Brodmann's area 11 receives information from olfactory input as well as activation of the mediodorsal nucleus of the thalamus (thought to be associated with episodic memory). Olfaction may be represented by an episodic memory attached to the specific odor. This suggests examining a crucial, but neglected paradigm by studying olfaction by looking at higher-level representation such as episodic memory.

If one is to theorize about higher-level representation, it is important to establish that said theory is built upon a plausible neurological model. The projection of olfactory impulses into the limbic system helps support the idea that a significant effect of emotion could interact with the relationship between memory and olfaction. Furthermore, there is a projection from the limbic system into the prefrontal cortex (area associated with episodic memory). This projection, known as Papez (1937) circuit is a collection of neural connections shows a possible route of connection between olfaction and episodic memory through the emotional center of the brain.

In this study we will examine the role of possible emotional and olfactory influences in episodic memory. Specifically, we will examine if the Proust phenomenon of olfaction will generalize to non-autobiographical memory for vignettes. Comparing episodic memory for vignettes to autobiographical episodic memory is a matter of self-relevance. Autobiographic episodic memory can be compared to the episodic memory of your own wedding. Non-autobiographical episodic memory (such as for vignettes) is similar to the episodic memory for reading a story about a wedding. If episodic memory is the code for the storage of olfaction, (Stevenson and Boakes, 2003) then the olfaction and episodic memory should be significantly affected by emotional content. This is because the neural pathways from olfactory receptors travel to areas of episodic memory through the limbic system. Work by Adolphs et al. (2003) gathered evidence for a neurological basis for the relationship between emotions and episodic memory via an associated body state. The data from the study supports the idea of a neurological basis of dissociable neural systems for emotions that are separate neural systems and are specific to an emotion (Adolphs et al., 2003). This diversity of systems could facilitate emotion as a somatic marker.

The role of the ventromedial stream in emotion is quite remarkable and supports emotion's role as a somatic marker. Tranel (2002) discussed the concept of a gambling task involving people with ventromedial prefrontal cortex lesions using this gambling task. Presumably, in the gambling task, decision making comes from some minor emotional response to a particular deck. This theoretical "gut reaction" is also known as a somatic marker hypothesis which was proposed by Damasio (1994). The idea is the neural firing to the ventromedial stream triggers a slight emotional reaction which serves as a somatic marker as to whether one would like to use a particular deck of cards or not and switch over to another more profitable deck. What has been found in work involving people with the ventromedial lesion is that they perform more poorly on this task and it is more likely due to an inability to receive a somatic marker to understand when the time has come to switch. This work has been very relevant, especially in lines of the idea of using a somatic marker hypothesis for the works of Stevenson and Boakes (2003) in the idea that the olfactory cue is a somatic marker for episodic memory and can explain more of the Proust phenomenon of olfaction. The demonstration of olfactory cuing benefits rests on showing a connection to improved episodic memory performance through emotional mediation. In contrast to previous studies that examined autobiographical episodic memory, we will look at the role of olfaction and emotion in memory for non-autobiographical material.

Hypothesis: Due to the Proust phenomenon of autobiographical episodic memory; our hypothesis is that a relationship exists between non-autobiographical episodic memory and olfaction. Because of the position of the limbic system in the neural architecture of olfaction and episodic memory, it is hypothesized that emotional context should improve performance on nonautobiographical memory for vignettes when coupled with olfactory stimuli. This hypothesis would be supported by evidence of enhanced memory performance in the presence of olfactory cues and emotional context relative to memory performance in the absence of olfactory cues and/or emotional context.

\section{MATERIALS AND METHODS}

\section{Experiment 1:}

Participants: Participants in this experiment were 36 adults ( 24 female and 12 male; mean age $=22.7$ years) recruited from University of Akron undergraduate 
psychology classes. Participants were compensated with credit towards their class grade. All participants were native English speakers and reported no hearing difficulty. Participants evaluated their sense of smell on a 7 point Likert scale $(1=$ worst and $7=$ best $)$ with a mean of 5.2.

Vignettes: Six vignettes were used in this experiment. Three vignettes contained material of an emotional nature, while the other 3 were emotionally neutral. All vignettes were presented aurally. A recording was made of a male voice reciting the vignettes and presented through computer speakers. This had the merit of making the aural presentations to all subjects as equal as possible in terms of exposure duration. A key area of interest is that the emotional content of the vignettes is not directly stated in the vignettes. For example, when one listens to a vignette about anger, there is no direct use of the word anger or any synonym of anger.

Emotional vignettes contained stories that had extreme emotional content (i.e., a boy who died of cancer). Emotionally neutral vignettes contained stories that did not contain extreme emotional content (i.e., a man who has a collection of movie memorabilia). All vignettes were matched to be a close in length and detail as possible.

Olfactory stimuli: The odor stimuli used were presented each in a separate ceramic vial which the subjects held approximately $7 \mathrm{~cm}$ under their noses. They were instructed to breathe normally and listen to each vignette. In all cases in which there was the presentation of an odor stimulus, the same stimulus was used at the time of testing (if tangerine scent was presented with the first vignette then the participant would receive the tangerine scent when answering questions about the first vignette). There were 3 odor stimuli: tangerine extract, bergamot extract and sulfur.

Procedure: There were two phases of this experiment: exposure and testing. Half of the participants heard only the 3 emotional vignettes and the other half heard only the emotionally neutral vignettes. One third of the participants in each group (emotion or neutral) received an odor stimulus at the time of encoding and testing. One third received an olfactory cue at the time of encoding only. Finally, one third received no olfactory cue at either encoding or testing.

There were six groups related to emotional content and olfactory stimulus. Emotion-Cue-Cue received emotional vignettes and had olfactory stimuli present at the time of vignette presentation and at the time of testing. Emotion-Cue-No Cue had emotional vignettes and received an olfactory stimulus at the time of vignette presentation, but not at the time of testing. Neutral-Cue-Cue had emotionally neutral vignettes and olfactory stimuli present for both vignette presentation and time of testing. Neutral-Cue-No Cue had emotionally neutral passages at the time of presentation and not time of testing. Emotion-No Cue-No Cue had emotional vignettes only with no olfactory stimuli at both times. Neutral-No Cue-No Cue had emotionally neutral passages and received no olfactory stimuli at any time.

The odors were counterbalanced across all vignettes in the olfaction groups. For Emotion-Cue-Cue and Neutral-Cue-Cue groups, no olfactory cue was given for a test over a vignette that did not correspond to the odor-vignettes pair at time of encoding. For example, if a subject receives exposure to tangerine extract during the presentation of vignettes $A$, then at time of testing they receive tangerine extract as a cue for the test of comprehension for vignettes A. At no time was there a switching of cue to vignettes pairing between exposures and testing.

After each vignettes exposure a quick self-report measure was given of the subject's countenance towards the vignettes and odor (if presented). After a period of distraction during which demographics were taken (approximately $3 \mathrm{~min}$ ) memory tests were given to participants over the content of the vignettes that were presented earlier. Each memory asked open ended questions to the respective vignettes such as "How old was Aunt Nancy?___ or "Was there any coffee available that morning?___.

Results: An Analysis Of Variance (ANOVA) was performed on the data from Experiment 1. Results are presented in Table 1. There was a significant effect for group $\mathrm{F}(5,30)=10.75, \mathrm{p}>0.001$. A Tukey's HSD test revealed significant differences between Emotion-CueCue and Neutral-Cue-Cue $(p<0.001)$, Emotion-CueCue and Neutral-Cue-Cue $(\mathrm{p}<0.001)$, Emotion-CueCue and Neutral-No Cue-No Cue $(\mathrm{p}<0.001)$, EmotionCue-No Cue and Neutral-Cue-Cue $(p=0.024)$ and Neutral-Cue-No Cue and Emotion-No Cue-No Cue $(\mathrm{p}=0.003)$. The significant differences between Emotion-Cue-Cue and Neutral-Cue-Cue, Neutral-CueCue and Neutral-No Cue-No Cue indicated better recall of emotional passages with an accompanying olfactory cue than neutral passages alone (regardless of whether the neutral passage had an olfactory cue). 
Table 1: Mean percentage recall (\%) and standard deviation for experiment 1

\begin{tabular}{llr}
\hline Group & Mean & SD \\
\hline Emotion-Cue-Cue & 55.00 & 15.126 \\
Emotion-Cue-No Cue & 38.00 & 11.027 \\
Neutral-Cue-Cue & 26.00 & 8.270 \\
Neutral-Cue-No Cue & 18.17 & 8.750 \\
Emotion-No Cue-No Cue & 42.83 & 8.886 \\
Neutral-No Cue-No Cue & 25.00 & 6.899 \\
Overall mean & 34.17 & 15.760 \\
\hline
\end{tabular}

The finding of significant difference between EmotionCue-No Cue and Neutral-Cue-Cue suggested that although both passages received an olfactory stimulus at the time of encoding only; there was a significant advantage of emotional content over emotionallyneutral content. A significant difference between Neutral-Cue-Cue and Emotion-No Cue-No Cue suggested better recall for emotional passages alone than for neutral passages that had been exposed to an olfactory stimulus at the time of encoding only. The Tukey's HSD also revealed marginally significant differences between Emotion-No Cue-No Cue and Neutral-No Cue-No Cue $(p=0.051)$, Neutral-Cue-Cue and Emotion-No Cue-No Cue $(\mathrm{p}=0.076)$ and Emotion-Cue-Cue and Emotion-Cue-No Cue ( $\mathrm{p}=$ 0.068 ).

In an Analysis Of Covariance (ANCOVA) we found that there was a significant effect of group when controlling for subjects' reported emotional experience of the vignettes, $F(5,29)=4.58, p<0.05$. Furthermore, a pairwise comparison shows a significant effect of Emotion-Cue-Cue over Emotion-Cue-No Cue $(\mathrm{p}<0.05)$, Neutral-Cue-Cue $(\mathrm{p}<0.01)$, Neutral-Cue-No Cue $(\mathrm{p}<0.01)$, Emotion-No Cue-No Cue $(\mathrm{p}<0.05)$ and Neutral-No Cue-No Cue $(\mathrm{p}<0.01)$.

Discussion: Experiment 1: The results of Experiment 1 showed that emotional content with an olfactory cue at the time of testing promoted better recall than neutral passages (with and without olfactory cues). Furthermore, the results supported other studies by showing that emotional content produced better recall performance than emotionally-neutral content. Experiment 1 demonstrates a clear beneficial effect of pairing an olfactory cue to an emotional passage on a test of nonautobiographical memory. Furthermore there is a benefit to recall for passages with emotional content as opposed to passages that are emotionally neutral. However, the role of olfaction in assisting recall for emotional passages over emotional passages without olfactory cues remains somewhat unclear.

We see a marginally significant benefit to having the olfactory cue present at encoding and recall as opposed to having the olfactory cue at encoding only when using emotional passages. However, there was no significant (only marginally) difference between emotional passages only and emotional passages with an olfactory cue. There are several possible explanations for this. The first is that emotion is a more salient factor in recall than olfaction. The results of the analysis of covariance suggest that when accounting for emotional rating the effect of emotion and olfactory cues as both encoding and retrieval increase performance above emotion alone. Many studies (Kensinger et al., 2007) indicate the important role emotion plays in memory. This would suggest that emotion is a primary cue and sensory cues (olfaction) may be secondary compared to emotion. Another explanation is that the olfactory cues may have been connected to the vignettes in a superficial way. The goal of experiment 2 was to test the latter explanation by incorporating olfaction into the vignettes.

\section{Experiment 2:}

Participants: Participants in this experiment were 40 adults (31 female and 9 male; mean age $=22.5$ years) recruited from Plattsburgh State University undergraduate psychology classes. Participants were compensated with credit towards their class grade. All participants were native English speakers and reported no hearing difficulty. Participants evaluated their sense of smell on a 7 point Likert scale $(1=$ worst and $7=$ best) with a mean of 5.1.

Experiment 2 utilized modified vignettes from Experiment 1 . The vignettes were modified so that each vignette mentioned a specific odor twice within each vignette. In the vignettes an odor was be mentioned, but not by the name of the odor presented. For example a vignette might mention the smell of a forest while the subject is exposed to a potpourri smell (bergamot extract), but the vignette never mentions that the smell was potpourri nor did the experimenter describe the potpourri smell (bergamot extract) as a "forest scent." All odors were paired with the vignettes that specifically mentioned the quality of the olfactory stimulus (example, sulfur was not paired with the vignette that refers to tangerine).

Procedure: Procedures were identical to Experiment 1. There were four groups only for Experiment 2. Emotion-Cue received emotional vignettes with olfactory stimuli at time of vignette presentation and time of testing. Neutral-Cue received emotionallyneutral vignettes with olfactory stimuli at both time of presentation and time of testing. Emotion-No Cue received emotional vignettes and no olfactory stimuli at 
either time of presentation or time of testing. NeutralNo Cue received emotionally neutral vignettes and no olfactory stimuli at either time.

\section{RESULTS}

Data from Experiment 2 was used in an Analysis Of Variance (ANOVA). Results are presented in Table 2. There was a significant effect for group $F(3,36)=$ 9.84, $\mathrm{p}<0.001$. A Tukey's HSD revealed that there was a significant difference between Emotion-Cue and Neutral-Cue $(p<0.001)$, Emotion-Cue and Neutral-No Cue $(p=0.014)$. There was also a significant difference between Emotion-No Cue and Neutral-Cue $(p=0.001)$ as well as between Emotion-No Cue and Neutral-No Cue $(p=0.026)$. However there was no significant difference between Emotion-Cue and Emotion-No Cue (Fig. 2).

A follow-up Analysis Of Covariance (ANCOVA) did show a significant effect for group when the effect of subjects reported reaction was considered, $\mathrm{F}(3$, $16)=15.6, p<0.01$. A pairwise comparison failed to show a significant difference between Emotion-Cue (emotion and olfactory cue) and Emotion-No Cue (emotion cue only).

Table 2: Mean percentage recall $(\%)$ and standard deviation for experiment 2

\begin{tabular}{lrl}
\hline Group & Mean & SD \\
\hline Emotion-Cue & 17.91 & 4.721 \\
Neutral-Cue & 7.10 & 6.574 \\
Emotion-No Cue & 18.11 & 3.621 \\
Neutral-No Cue & 10.90 & 5.547 \\
Overall mean & 13.50 & 6.936 \\
\hline
\end{tabular}

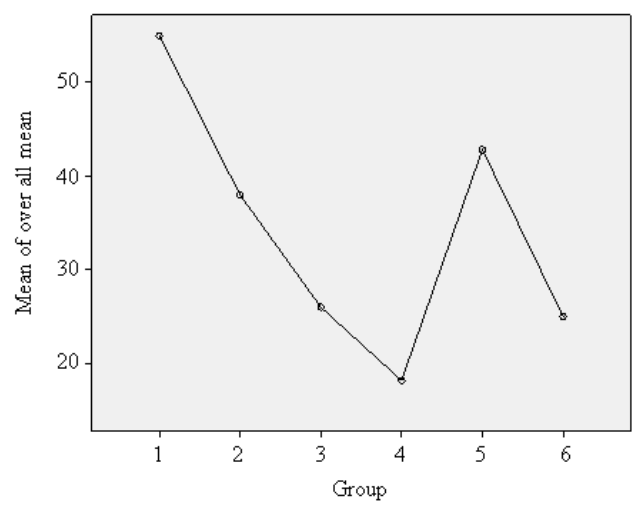

Fig. 1: Mean percentage correct for each group for Experiment 1 Group 1 = Emotion-Cue-Cue, Group 2 = Emotion-Cue-No Cue, Group $3=$ Neutral-Cue-Cue, Group 4 = Neutral-Cue-No Cue, Group 5 = Emotion-No Cue-No Cue, Group 6 = Neutral-No Cue-No Cue
Discussion: Experiment 2: Experiment 2 was conducted to examine if the effect of olfaction as a cue in emotional passages would be enhanced if olfactory stimuli were incorporated into the passages. The results of Experiment 2 replicated the findings of Experiment 1 concerning the effect of emotional content (Fig. 1). Participants had better recall for passages that contained emotional content over passages that were emotionally neutral.

However, there was no significant benefit (not even marginally) for emotional passages that were presented with an olfactory stimulus over passages that did not have an olfactory stimulus presented. These results suggest that although olfactory cues can be used in recall which are in line with previous research on olfaction and higher cognitive abilities (Danthiir et al., 2001) their role may be overshadowed by the emotional content of passages. Although olfactory cues can be used as a cue (as in Experiment 1) in episodic memory, emotion may be the more preferred salient feature of memory. Emotional content is often a defining feature such as in flashbulb memories. Flashbulb memories (Talarico and Rubin, 2007) are vivid memories noted for being memories of events associated with powerful emotions such as the attacks of September 11th, 2001 and the assassination of Benazir Bhutto. An olfactory cue may trigger and assist the recall of a memory, but emotion appears to be a more salient and powerful tool for recall.

The key contrast between Experiment 1 and 2 was that in Experiment 2 specifically mentioned an olfactory odor in each vignette. Stimuli not specifically mentioned may be encoded as context cues.



Fig. 2: Mean percentage correct for each group for Experiment 2. Group $1=$ Emotion-Cue, Group 2 $=$ Neutral-Cue, Group 3 = Emotion-No Cue, Group 4 = Neutral-No Cue 
However, when the odor is specifically mentioned, instead of being a context cue, it could become a semantic element or detail of the memory. This may cause olfactory cues to become less effective when mentioned specifically by making them less of a context cue and more of an imbedded fact within the vignette.

\section{DISCUSSION}

The two experiments were conducted to examine the roles emotion and olfaction in recall of episodic passages. We sought to expand on the Proust phenomenon of olfaction in episodic memory. It was hypothesized in this study that emotion and olfaction would both serve to enhance memory performance for vignettes.

Both experiments clearly demonstrated a benefit of emotional content in recall. Experiment 1 did show a marginally significant benefit for recall of emotional passages presented with an olfactory stimulus at encoding and at testing (Emotion-Cue-Cue) over having the olfactory stimulus at encoding only (Emotion-CueNo Cue). Experiment 2 did not show a significant effect for olfaction for emotional or neutral passages. The difference between Experiment 1 and 2 occurred despite specifically mentioning olfactory stimuli in the passages in Experiment 2.

The results of Experiment 2 suggest that the benefit for olfactory cues is minimized by the emotional content of passages. Furthermore, it was shown that the olfactory benefit is not to be found in emotionally neutral passages. It can be implied as from Experiment 1 that emotion and olfaction do produce better recall. However the contribution of olfaction may not be as important as previously thought, at least for nonautobiographical episodic memory. Indeed, a recent study (Zhou and Chen, 2009) has shown that olfactory stimuli can give emotional cues to ambiguous faces. Here we can see an overlap with processing of ambiguous faces (which can lead to less clarity about emotional state) with olfaction. Olfaction is still an important cue, but not in a state relatively absent of emotional context.

This study has some theoretical implications about the ability to generalize the Proust phenomenon of olfaction (Chu and Downes, 2000). When observing olfaction as a sensory cue for memory outside of an autobiographical context, we see that olfaction is not as salient as emotion. Most research on sensory cues in memory is didactic; with vision and audition being the preferred sensory mediums of interest. This study is one of the few to look beyond vision and audition to examine what role the other senses have in cognition and memory.

It is worth noting that the study of olfaction has many extraneous variables (Kobus Maree, personal communication, January 22, 2009). Some of the limitations of our method stem from difficulties in manipulating olfaction in an experimental setting. Although we asked participants to rate their response to the olfactory stimuli, there was no feasible way (for these experiments) to measure neurological activation of olfactory receptors. Another issue is the use of vignettes when we manipulated emotion that could also manipulate the context.

\section{CONCLUSION}

Most of the research on olfaction and memory (Chu and Downes, 2000) has focused on the role of olfaction in autobiographical episodic memory. This study examined episodic memories of passages. The extent of the benefit of olfaction on autobiographical episodic memory may not generalize to other episodic memory tasks as readily. An important direction for future studies will be to examine what other aspects of memory may or may not be affected by the interaction of emotion and olfaction.

\section{REFERENCES}

Adolphs, R., D. Tranel and A.R. Damasio, 2003. Dissociable neural systems for recognizing emotions. Brain Cogn., 52: 61-69. http://www.ncbi.nlm.nih.gov/pubmed/12812805

Brand, G., J.L. Millot and D. Henquell, 2001. Complexity of olfactory lateralization processes revealed by functional imaging: A review. Neurosci. Biobehav. Rev., 25: 159-166. PMID: 11323080

Chu, S. and J.J. Downes, 2000. Long live proust: The odour-cued autobiographical memory bump. Cognition, 75: 41-50. PMID: 10771279

Damasio, A.R., 1994. Descartes' Error: Emotion, Reason and the Human Brain. 1st Edn., Grosset/Putnam, New York, ISBN: 0399138943, pp: 312.

Danthiir, V., R.D. Roberts, G. Pallier and L. Stankov, 2001. What the nose knows: Olfaction and cognitive abilities. Intelligence, 29: 337-361. DOI: 10.1016/S0160-2896(01)00061-7

Doty, R.L., R. Smith, D.A. McKeown and J. Raj, 1994. Tests of human olfactory function: Principal components analysis suggests that most measure a common source of variance. Percep. Psychophys., 56: 701-707. PMID: 7816540 
Engen, T. and B.M. Ross, 1973. Long-term memory of odors with and without verbal descriptions. J. Exp. Psychol., 100: 221-227. DOI: 10.1037/h0035492

Ganong, W.F., 1999. Review of Medical Physiology. 19th Edn., Stamford, Connecticut, ISBN: 0838582524 , pp: 851.

Jehl, C., J.P. Royet and A. Holley, 1995. Odor discrimination and recognition memory as a function of familiarization. Percept. Psychophys., 57: 1002-1011. PMID: 8532490

Kensinger, E.A., J.L. O'Brian, K. Swanber, R.J. Garoff-Eaton and D.L. Schacter, 2007. The effects of emotional content on reality-monitoring performance in younger and older adults. Psychol. Aging, 22: 752-764. DOI: $10.1037 / 0882-7974.22 .4 .752$

Malmberg, K.J. and R.M. Shiffrin, 2005. The "oneshot" hypothesis for context storage. J. Exp. Psychol. Learn. Memory Cogn., 31: 322-336. http://www.ncbi.nlm.nih.gov/pubmed/15755248

Paivio, A., 1971. Imagery and Verbal Processes. 2nd Edn., Holt, Rinehart and Winston, New York, pp: 596.

Papez, J.W., 1937. A proposed mechanism of emotion. Arch. Neurol. Psychiatry, 38: 725-743. PMID: 7711480

Parker, A., H. Ngu and H.J. Cassaday, 2001. Odour and proustian memory: Reduction of context-dependent forgetting and multiple forms of memory. Applied Cognit. Psychol., 15: 159-171. DOI: 10.1002/1099720(200103/04)15:2<159::AID-

ACP694>3.0.CO;2-D
Pinel, J.J.P., 1990. Biopsychology. 1st Edn., Allyn and Bacon, MA., ISBN: 0205120520, pp: 576.

Stevenson, R.J. and R.A. Boakes, 2003. A mnemonic theory of odor perception. Psychol. Rev., 110: 340-364. PMID: 12747527

Talarico, J.M. and D.C. Rubin, 2007. Flashbulb memories are special after all in phenomenology, not accuracy. Applied Cogn. Psychol., 21: 557-578. DOI: $10.1002 / \mathrm{acp} .1293$

Tranel, D., 2002. Emotion, Decision Making and the Ventromedial Prefrontal Cortex. In: Principles of Frontal Lobe Function, Stuss, D.T. and R.T. Knight (Eds.). Oxford University Press, London, ISBN: 10: 0195134974, pp: 338-352.

Tulving, E., 1974. Recall and recognition of semantically encoded words. J. Exp. Psychol., 102: 778-787. DOI: 10.1037/h0036383

Yamaguchi, M., N. Tonoiki, I. Hirata, I. Kaetsu and R. Seo et al., 2001. Olfactory magnetic responses of right and left brain hemisphere in the oddball paradigm. Proceeding of the Meeting of BioMag (BM'01), Espoo, Finland, pp: 1-4. http://citeseerx.ist.psu.edu/viewdoc/download?doi= 10.1.1.5.330\&rep=rep1\&type $=$ pdf

Zhou, W. and D. Chen, 2009. Fear-related chemosignals modulate recognition of fear in ambiguous facial expressions. Psychol. Sci., 20: 177-183. PMID: 19170944 